\title{
Fermion Self-Energy and Chiral Symmetry Breaking from Four-Fermion and Gauge Interactions*
}

\author{
Bang-Rong Zhou \\ Department of Physics, The Graduate School at Beijing \\ University of Science and Technology of China, Academia Sinica \\ Beijing 100039, China ${ }^{\dagger}$ \\ and \\ CCAST ( World Laboratory ) P.O.Box 8730, Beijing 100080, China
}

\begin{abstract}
The exact analytic solutions of the linearized Schwinger-Dyson equation of fermion self-energy are used to obtain the effective four-fermion and gauge coupling criticality curves for dynamical chiral symmetry breaking. The results show that when the zero-momentum gauge coupling $\alpha(0)<\alpha_{0}(0)$, the critical gauge coupling in the pure gauge interaction case, the minimal critical four-fermion coupling $\beta_{\min }$ is always non-zero and positive and will go up as the $\alpha(0)$ decreases. The use of the exact solutions also allow us to make quite definite estimations of the momentum scales where chiral symmetry breaking would happen if the values of an infrared parameter $\xi$ are given separately.
\end{abstract}

PACS numbers: 12.38.Aw, 12.60.Nz, 11.30.Rd, 11.30.Qc

Key words: Fermion self-energy, Schwinger-Dyson equation, Chiral symmetry breaking, Criticality curve of four-fermion and gauge coupling

It is an interesting problem to determine the critical coupling constants and the momentum scales where chiral symmetry breaking will occour in a theory with effective four-fermion and gauge interactions. It has not only fundamental theoretical significance but also can find its important application in Technicolor(TC) [1], Extended Technicolor (ETC) [2] and the Top-quark condensate theory [3-6] of dynamical electroweak symmetry breaking. An efficient approach to persue this problem is to use the Schwinger-Dyson(SD) equation of fermion self-energy [7-13,4]. When effective four-fermion interactions are included in, different from the case of pure gauge interactions, one must introduce a finite momentum cut-off $\Lambda$ and this will produce an important impact on results. For instance, because the four-fermion coupling constant is defined at the scale $\Lambda$, the chiral symmetry breaking must much more depend on the ultraviolet (UV) asymptoticality of theory. On the other hand, in order to obtain some more quantitative predictions of the scales of chiral symmetry breaking we need the exactest possible solutions of the S-D equation rather

*The project supported partially by National Natural Science Foundation of China and by Grant No.LWTZ-1298 of the Chinese Academy of Sciences.

${ }^{\dagger}$ Mailing address. 
than only the UV asymptotic form of these solutions. Fortunately, we have obtained the exact analytic solutions of the S-D equation of fermion self-energy in some linearization approximation [14]. In this paper, we will use these solutions to discuss dynamical chiral symmetry breaking under a finite momentm cut-off.

The Lagrangian of the system to be delt with may be described by

$$
\mathcal{L}=-\frac{1}{4} F_{\mu \nu}^{a} F^{a \mu \nu}+\bar{\psi} i \gamma^{\mu}\left(\partial_{\mu}+i g A_{\mu}^{a} \lambda^{a}\right) \psi+h\left[(\bar{\psi} \psi)(\bar{\psi} \psi)-\left(\bar{\psi} \gamma_{5} \psi\right)\left(\bar{\psi} \gamma_{5} \psi\right)\right]
$$

where $\psi$ is the bare-massless fermion field which is assigned in the representation $R_{\psi}$ of the color gauge group $G$ with dimention $d\left(R_{\psi}\right), A_{\mu}^{a}$ and $F_{\mu \nu}^{a}$ are respectively the gauge field and corresponding field strength tensor, $\lambda^{a}$ is the generator of the gauge group $G, g$ and $h$ are respectively the coupling constant of the gauge and the chirally invariant fourfermion interactions. It has been well known $[11,4,14]$ that, the integral S-D equation of the fermion self-energy $\Sigma(x)$ in the ladder approximation and in the Landau gauge can be reduced to the differential equation

$$
\omega(x) \Sigma^{\prime \prime}(x)+\left[\omega^{\prime}(x)+1\right] \Sigma^{\prime}(x)=-\frac{b}{\tau(x)} \frac{\Sigma(x)}{\left[x+\Sigma^{2}(x)\right]}
$$

together with the IR boundary condition

$$
\Sigma^{\prime}(0)=-\frac{b}{2(\ln \xi) \Sigma(0)}
$$

and the UV boundary condition

$$
\left\{\left[1+\frac{\beta}{b} \tau(x)\right] \omega(x) \Sigma^{\prime}(x)+\Sigma(x)\right\}_{x=\Lambda^{2}}=0
$$

where $x=p^{2}$ is the squared Euclidean four-momentum and $\Lambda$ is the momentum cut-off. The constant

$$
b=\frac{3 C_{2}\left(R_{\psi}\right)}{16 \pi^{2} \beta_{0}}
$$

with the eigenvalue $C_{2}\left(R_{\psi}\right)$ of the squared Casimir operator of the gauge group $G$ in the fermion field representation $R_{\psi}$ and the coefficient of the $\beta$-function of the gauge coupling $g$ to one loop order

$$
\beta_{0}=\left[11 C_{2}(G)-\sum_{f} 4 T\left(R_{f}\right) N_{f}\right] / 48 \pi^{2}
$$

where the standard denotations in gauge theory have been used and in the flavor sum $\sum_{f}$ in Eq.(6), the $\psi$ fermion and all the lighter colored fermion flavors in the $G$-representation $R_{f}$ will be included in. In the derivation of Eqs.(2)-(4) a continuous Ansatz [11] of the running gauge coupling constant

$$
\bar{g}^{2}(x)=1 / \beta_{0} \tau(x)
$$

with

$$
\tau(x)=\ln \left(\frac{x}{\mu^{2}}+\xi\right)
$$

has been used. The scale parameter $\mu$ and the IR parameter $\xi$ are optional except that $\xi>1$ is required so as to avoid the IR singularity of $\bar{g}^{2}(x)$. The constant $\beta$ is connected to the strength of the four-fermion interactions and defined by

$$
\beta=\frac{d\left(R_{\psi}\right) h \Lambda^{2}}{2 \pi^{2}}
$$


It is noticed that $\beta$ appears only in the UV boundary condition (4), not in the equation (2). The function $\omega(x)$ is defined by

$$
\omega(x)=\left[\frac{1}{x}+\frac{1}{\left(x+\xi \mu^{2}\right) \tau(x)}\right]^{-1}
$$

The linearization approximation of Eq.(2) means that [14]

1) the $\Sigma^{2}(x)$ in the denominator of the right-handed side of Eq.(2) will be replaced by the assumption

$$
\Sigma^{2}(0)=\xi \mu^{2}
$$

2) the function $\omega(x)$ will be substituted by its approximate expression

$$
\omega(x) \simeq \frac{\tau(x)}{1+\tau(x)}\left(x+\xi \mu^{2}\right)
$$

so that Eq.(2) could be solved exactly. The assumption (11) is permissible since the parameters $\xi$ and $\mu$ are both undetermined theoretically. The approximation (12) is valid if

$$
\frac{x}{\xi \mu^{2}}\left[1+\frac{1}{\ln \left(\frac{x}{\mu^{2}}+\xi\right)}\right] \gg 1
$$

and it is certainly satisfied when $x \gg \xi \mu^{2}$. In the following we will also extend the solutions of the linearized equation down to $x=\xi \mu^{2}$ as a further approximation.

In this way, the S-D equation (2) can be changed into that

$$
\frac{\tau}{1+\tau} \Sigma^{\prime \prime}(\tau)+\left[1+\frac{1}{(1+\tau)^{2}}\right] \Sigma^{\prime}(\tau)+b \frac{\Sigma(\tau)}{\tau}=0
$$

Eq.(14) may have exact analytic solution with the general expression

$$
\Sigma(\tau)=A \Sigma_{\text {irreg }}(\tau)+B \Sigma_{\text {reg }}(\tau)
$$

where $A$ and $B$ are two real constants and $\Sigma_{\text {irreg }}(\tau)$ and $\Sigma_{\text {reg }}(\tau)$ are two linearily independent solutions of Eq.(14)

$$
\left.\left.\begin{array}{l}
\Sigma_{\text {irreg }}(\tau) \\
\Sigma_{\text {reg }}(\tau)
\end{array}\right\}=\begin{array}{c}
A_{i} \\
A_{r}
\end{array}\right\} M(\tau)+\text { c.c. }
$$

where

$$
A_{i}=i(-1)^{\alpha} \frac{\Gamma(\bar{\alpha})}{\Gamma(\gamma)} \frac{\sin (\pi \bar{\alpha})}{\sinh (2 \pi \eta)}, \quad A_{r}=-i \frac{\pi}{b \sinh (2 \pi \eta)|\Gamma(\bar{\alpha})|^{2}} \frac{\Gamma(\bar{\alpha})}{\Gamma(\gamma)}
$$

and

$$
M(\tau)=e^{-\tau} \tau^{-\frac{1}{2}-i \eta}\left[\frac{\gamma}{2}{ }_{1} F_{1}(\bar{\alpha} ; \gamma ; \tau)+\frac{\bar{\alpha}}{\gamma}{ }_{1}{ }_{1} F_{1}(\bar{\alpha}+1 ; \gamma+1 ; \tau)\right]
$$

with the denotations

$$
\begin{aligned}
\eta & =\sqrt{b-\frac{1}{4}} \\
\alpha & =-\frac{1}{2}+b-i \eta \\
\bar{\alpha} & =\frac{1}{2}-b-i \eta \\
\gamma & =1-i 2 \eta
\end{aligned}
$$


and that $\Gamma(\bar{\alpha})$ is Gamma function and ${ }_{1} F_{1}(\bar{\alpha} ; \gamma ; \tau)$ is confluent hypergeometric (Kummer) function. When $\tau \rightarrow \infty$, the solutions (16) will approach their UV asymptotic forms [9]:

$$
\begin{aligned}
& \Sigma_{\text {irreg }}(\tau) \stackrel{\tau \rightarrow \infty}{\rightarrow} \tau^{-b} \\
& \Sigma_{\text {reg }}(\tau) \stackrel{\tau \rightarrow \infty}{\rightarrow} e^{-\tau} \tau^{b-1}
\end{aligned}
$$

However, it is emphasized that in the case with the finite momentum cut-off $\Lambda$ owing to presence of the four-fermion interactions, the distinquishment between $\Sigma_{\text {irreg }}(\tau)$ and $\Sigma_{\text {reg }}(\tau)$ is not important and one must consider the general solution (15) as a linear combination of the two independent solutions, i.e. normally both $A \neq 0$ and $B \neq 0$.

The solution (15) based on the approximation (12) is obviously inapplicable in the region $x<\xi \mu^{2}$. Therefore, we must impose the IR boundary condition of the solution at $x=\xi \mu^{2}[14]$

$$
\frac{d}{d \tau} \Sigma(\tau)=\left.b\left(\frac{d \tau}{d x}\right)^{-1} \frac{d}{d x}\left[\frac{1}{x \tau(x)}\right] \int_{0}^{x} d y \frac{y \Sigma(y)}{y+\Sigma^{2}(y)}\right|_{x=\xi \mu^{2}}
$$

where the expression of $\Sigma(x)$ in $x<\xi \mu^{2}$ can be approximated by the solution of Eq.(2) at small $x$, i.e.

$$
\begin{gathered}
\Sigma(x)=\Sigma(0)\left\{1-\frac{b}{2(\ln \xi) \Sigma^{2}(0)} x+\frac{b}{6(\ln \xi) \Sigma^{4}(0)}\left(1-\frac{b}{2 \ln \xi}\right) x^{2}\right. \\
\left.-\frac{b}{12(\ln \xi) \Sigma^{6}(0)}\left[1-\frac{5 b}{3 \ln \xi}+\frac{b^{2}}{3 \ln ^{2} \xi}\right] x^{3}+\cdots\right\} \quad \text { for } x<\xi \mu^{2}
\end{gathered}
$$

It is indicated that the solution (22) submits the IR boundary condition (3) at $x=0$. Considering the assumption (11) and the fact that $\Sigma(x)$ must be continuous function at $x=\xi \mu^{2}$ we may change Eq.(21) into that

$$
\Sigma^{\prime}\left(\tau_{1}\right)=-P\left(\tau_{1}\right) \Sigma\left(\tau_{1}\right), \quad \tau_{1}=\ln (2 \xi)
$$

where

$$
\begin{gathered}
P\left(\tau_{1}\right)=b \frac{2 \tau_{1}+1}{\tau_{1}^{2}} \frac{1}{f(1)} \int_{0}^{1} d t \frac{t f(t)}{t+f^{2}(t)} \\
f(t)=1-\frac{b}{2 \ln \xi} t+\frac{b}{6 \ln \xi}\left(1-\frac{b}{2 \ln \xi}\right) t^{2}-\frac{b}{12 \ln \xi}\left(1-\frac{5 b}{3 \ln \xi}+\frac{b^{2}}{3 \ln ^{2} \xi}\right) t^{3}
\end{gathered}
$$

Now the whole problem becomes to seek the physical solution with the form (15) which also satisfies the UV and the IR boundary conditions (4) and (23). The UV boundary condition (4) can be rewritten and turned into that

$$
\beta=\frac{b}{\tau\left(\Lambda^{2}\right)}\left[-1-\frac{1+\tau\left(\Lambda^{2}\right)}{\tau\left(\Lambda^{2}\right)} \frac{\Sigma\left(\tau\left(\Lambda^{2}\right)\right)}{\Sigma^{\prime}\left(\tau\left(\Lambda^{2}\right)\right)}\right]
$$

It is pointed out that in view of the expression $(21)$ of $\Sigma^{\prime}(\tau)$, when taking off the gauge interactions i.e. setting $b=0$ we will have $\Sigma(x) \rightarrow m=$ constant and may obtain from Eq. (26) that

$$
\beta=\Lambda^{2} / \int_{0}^{\Lambda^{2}} d y \frac{y}{y+m^{2}}=1 /\left[1-\frac{m^{2}}{\Lambda^{2}} \ln \frac{\Lambda^{2}+m^{2}}{m^{2}}\right]
$$

which is just the gap equation of the Nambu-Jona-Lasinio (NJL) model [15] and now identical with the UV boundary condition. Denoting the strength of the running gauge coupling at the momentum cut-off scale $\Lambda$ by

$$
\alpha^{(\Lambda)} \equiv \frac{\bar{g}^{2}\left(\Lambda^{2}\right)}{4 \pi}=\frac{4 \pi b}{3 C_{2}\left(R_{\psi}\right) \tau\left(\Lambda^{2}\right)}
$$


we may obtain from Eqs.(15), (16) and (26) that

$$
\beta=\frac{b}{\tau}\left[-1-\frac{1+\tau}{\tau} \frac{\left(A_{i}+\frac{B}{A} A_{r}\right) M(\tau)+c . c .}{\left(A_{i}+\frac{B}{A} A_{r}\right) M^{\prime}(\tau)+c . c .}\right]_{\tau=\frac{3 C_{2}\left(R_{\psi}\right) \alpha(\Lambda)}{4 \pi b}}
$$

From the IR boundary condition (23), it may be found out that the real constant

$$
\frac{B}{A}=-\frac{A_{i}\left[M^{\prime}\left(\tau_{1}\right)+P\left(\tau_{1}\right) M\left(\tau_{1}\right)\right]+\text { c.c. }}{A_{r}\left[M^{\prime}\left(\tau_{1}\right)+P\left(\tau_{1}\right) M\left(\tau_{1}\right)\right]+\text { c.c. }}
$$

It depends upon the single infrared parameter $\xi$ when $b$ is given in a particular model. The value of $\xi$ can be fixed by phynomenology. However, here we prefer some general discussions about the results of its possible values. Besides $\xi>1$, a further theoretical constraint on $\xi$ may come from the requirement that $\Sigma(\tau)$, as mass function of the fermion, must be positive-definite. In particular, it must be so that

$$
\Sigma\left(\tau=\tau_{1}\right)>0
$$

which , by Eq.(22), will mean a new lower bound to be imposed on $\xi$.

Eq.(29) actually give the $\beta-\alpha^{(\Lambda)}$ criticality curve of chiral symmetry breaking for a general theory with effective four-fermion and gauge interactions. To make a concrete insight, we will apply it to a model based on one-generation of technifermions where we identify the $\psi$-field with one of the technifermion "flavors", the group $G$ with the technicolor gauge group $S U(4)$. In this case the constant

$$
b=\frac{135}{224}
$$

if the eight "flavors" of the one generation of technifermions are included in the calculation of $\beta_{0}$. It follows from Eq.(31) that the constraint on $\xi$ will be

$$
\xi>1.30017
$$

we find that $B / A$ is sensitive to the change of $\xi$ and has the following results:

$$
\frac{B}{A}\left\{\begin{array} { l } 
{ < 0 , } \\
{ > 0 } \\
{ < 0 , }
\end{array} ( \text { from } \infty \text { to } 0 ) , \text { if } \left\{\begin{array}{l}
1.30017<\xi<1.44043 \\
1.44043<\xi<3.09975 \\
3.09975<\xi<\infty
\end{array}\right.\right.
$$

We can obtain the further constraint on $\xi$ from $\Sigma(\tau)>0$ for all $\tau>\tau_{1}$. By using the continuity of $\Sigma(\tau)$ at $\tau=\tau_{1}$, we get from Eqs.(15) and (16) that

$$
\frac{\Sigma(\tau)}{\Sigma\left(\tau_{1}\right)}=\frac{\left(A_{i}+\frac{B}{A} A_{r}\right) M(\tau)+\text { c.c. }}{\left(A_{i}+\frac{B}{A} A_{r}\right) M\left(\tau_{1}\right)+\text { c.c. }} \quad \text { if } \tau \geq \tau_{1}
$$

Since it has been demanded that $\Sigma\left(\tau_{1}\right)>0$, the sign of $\Sigma(\tau)$ for $\tau>\tau_{1}$ will be determined by the sign of the right-handed side of Eq.(35) which depends on $\xi$. It is not difficult to verify that $\Sigma(\tau)$ will become negative for large enough $\tau$ if $1.30017<\xi<1.44043$. For example, $\Sigma(\tau)<0$ if $\xi=1.35$ and $\tau>1.6555$ and if $\xi=1.44$ and $\tau>7.92921$ respectively. Hence the corresponding $\Sigma(\tau)$ can not be physical solutions. On the other hand, if $\xi>1.44043$ then we will have $\Sigma(\tau)>0$ and $\Sigma^{\prime}(\tau)<0$ for all $\tau \geq \tau_{1}$ and the 
corresponding $\Sigma(\tau)$ could be physical solutions.

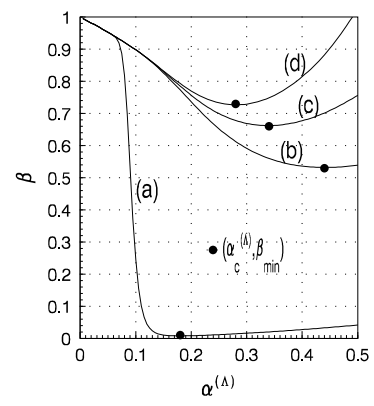

Fig. 1. The effective four-fermion and the gauge coupling $\beta$ and $\alpha^{(\Lambda)}$ criticality curves are depicted for (a) $\xi=1.44044(B / A \sim \infty)$; (b) $\xi=2(B / A=4.12519)$; (c) $\xi=3.09975\left(B / A=7.03327 \times 10^{-6} \sim 0\right)$ and $(\mathrm{d}) \xi=5(B / A=-2.58869)$.

Table 1. The numerical values of relevant parameters to the curves (a), (b), (c) and (d) in Fig. 1.

\begin{tabular}{ccccc}
\hline Curve & $(\mathrm{a})$ & $(\mathrm{b})$ & $(\mathrm{c})$ & $(\mathrm{d})$ \\
\hline$\xi$ & 1.44044 & 2 & 3.09975 & 5 \\
$B / A$ & 114865 & 4.12519 & $7.03327 \times 10^{-6}$ & -2.58869 \\
$\alpha_{1}$ & 1.27247 & 0.97122 & 0.737967 & 0.584733 \\
$\alpha_{c}^{(\Lambda)}$ & 0.179016 & 0.440915 & 0.338492 & 0.280842 \\
$\beta_{\min }$ & $7.71772 \times 10^{-3}$ & 0.532062 & 0.662304 & 0.726835 \\
$\Lambda_{c}(\mathrm{GeV})$ & - & 1078 & 1745 & 2647 \\
\hline
\end{tabular}

In Fig.1 we give the $\beta-\alpha^{(\Lambda)}$ curves for several typical values of $\xi$. The numerical values of relevant parameters to these curves are listed in Table 1 . In Table 1 the denotation $\alpha_{1}=4 \pi b / 3 C_{2}\left(R_{\psi}\right) \tau_{1}$ has been used and the momentum scales $\Lambda_{c}$ are found out from Eqs.(28), (8) and (32), $C_{2}\left(R_{\psi}\right)=15 / 8$ and the values of $\alpha_{c}^{(\Lambda)}$, if we assume the TC scale parameter $\mu=v=246 \mathrm{GeV}$, where $v$ is the vacuum expectation value of the Higgs field in the standard electroweak theory. For each $\beta-\alpha^{(\Lambda)}$ curve, we obtain a $\beta$ 's minimum point $\left(\alpha_{c}^{(\Lambda)}, \beta_{\min }\right)$ in the UV region whose location has been indicated explicitly by a dot in the curve. It is seen from Table 1 that $\alpha_{c}^{(\Lambda)}$ are always much less than $\alpha_{1}$. Since the solution (15) of Eq.(14) is reliable only in the region $x \gg \xi \mu^{2}$ i.e. $\tau \gg \tau_{1}$ or $\alpha^{(\Lambda)} \ll \alpha_{1}$, we will consider only the sectors of the $\beta-\alpha^{(\Lambda)}$ curves in the regions $\alpha^{(\Lambda)} \leq \alpha_{c}^{(\Lambda)}$ as physically acceptive criticality curves of the coupling constants.

The curve (a) shows that, when the value of $\xi$ is taken so that $B / A \rightarrow \infty$, the minimum point of $\beta$ will approach the origin, i.e. $\left(\alpha^{(\Lambda)}, \beta\right)=(0,0)$ becomes a critical point. This can be verified rigorously by taking $B / A \rightarrow \infty$ and $\alpha^{(\Lambda)} \rightarrow 0$ in Eq.(29). Since now $A \rightarrow 0$, we will leave only the regular term $\Sigma_{\text {reg }}(\tau)$ in Eq.(15) corresponding to the dynamical fermion mass generated by pure gauge interactions. In this case no finite momentum cut-off is needed because in an asymptotically-free gauge theory the running gauge coupling $\alpha^{(\Lambda)}=\frac{\bar{g}^{2}(\Lambda)}{4 \pi} \rightarrow 0$ can be attained in the limit $\Lambda \rightarrow \infty$. This result is coincided with the conclusion in Ref.[4]. By means of the present value $\xi=1.44044$ we may obtain the running gauge coupling at $p^{2}=0$ i.e. $\alpha_{0}(0)=3 \pi / 7 \ln \xi=3.689$, which, based on the analyses in Ref.[11], can be regarded as the critical gauge coupling for occurance of chiral symmetry breaking in the case of pure gauge interactions.

The curve (b) shows the general feature of $\beta-\alpha^{(\Lambda)}$ in a finite momentum cut-off $\Lambda$. Now we have both $A \neq 0$ and $B \neq 0$, corresponding to existence of both the irregular 
term $\Sigma_{\text {irreg }}(\tau)$ and the regular term $\Sigma_{\text {reg }}(\tau)$ in $\Sigma(\tau)$. We note that $\left(\alpha^{(\Lambda)}, \beta\right)=(0,1)$ is a critical point which represents the case without the gauge interactions and is just the NJL model. Then the critical curve $\beta$ becomes a monotonically decreasing function of $\alpha^{(\Lambda)}$ until $\alpha^{(\Lambda)}=\alpha_{c}^{(\Lambda)}=0.440915$ where $\beta$ is down to its minimum value $\beta_{\min }=0.532062$. This means that for $\beta<1$, we must have $\alpha^{(\Lambda)}>0$ in order to realize chiral symmetry breaking. It may also be concluded that if $\beta<\beta_{\text {min }}$ then chiral symmetry breaking could never happen. We indicate that in present case, $\xi=2$ hence the zero-momentum gauge coupling $\alpha(0)=3 \pi / 7 \ln 2=1.9424$ is less than the critical gauge coupling $\alpha_{0}(0)$ in tha case of pure gauge interactions and this can explain why we need a non-zero lower bound $\beta_{\text {min }}$ of the four-fermion coupling $\beta$ so that chiral symmetry breaking could occur only at $\beta>\beta_{\text {min }}$. A numerical estimation of the scales of chiral symmetry breaking can be made from the $\beta-\alpha^{(\Lambda)}$ criticality curve in the region $\alpha^{(\Lambda)} \leq \alpha_{c}^{(\Lambda)}$. It is obtained from the third column in Table 1 that if $\beta>\beta_{\min }$, then the corresponding scales of chiral symmetry breaking will be at $\Lambda>\Lambda_{c}=1078 \mathrm{GeV}$.

The curve (c) shows another extreme case where $B / A \rightarrow 0$, i.e. only the irregular term $\Sigma_{\text {irreg }}(\tau)$ is left in $\Sigma(\tau)$. The result remains to come from a combined effect of both the four-fermion and the gauge interactions. By comparing the curve (c) with the curve (b) we find that both the $\beta-\alpha^{(\Lambda)}$ criticality curves have the similar shape. A numerical check indicates that as the value of $\xi$ increases $\beta_{\text {min }}$ will always go up. This is plausible because the increase of $\xi$ will imply the decrease of the corresponding $\alpha(0)$, and this has to be compensated by rising of $\beta_{\min }$ so that chiral symmetry breaking could happen. We also note that when $\xi$ increases the corresponding $\alpha_{c}^{(\Lambda)}$ will arise at first but then come a continuative drop if $\xi>1.528$. This implies that for $\xi>1.528$, as $\xi$ increases we need stronger four-fermion interactions but weaker gauge interactions so as to realize dynamical chiral symmetry breaking. Consequently, a higher scale of chiral symmetry breaking is expected. This is shown in Table 1. For example, for $\xi=3.09975$ we need $\beta \geq \beta_{\min }=0.662304$ and the corresponding scales of chiral symmetry breaking will be $\Lambda \geq \Lambda_{c}=1745 \mathrm{GeV}$.

The curve (d) shows another different case where $B / A<0$, i.e. the irregular and the regular term of $\Sigma(\tau)$ have opposite sign but the whole $\Sigma(\tau)$ remains to be positive. In this case, the shape of the curve is still similar to the ones of the curve (b) and the curve (c) except that it has bigger $\beta_{\min }$ and smaller $\alpha_{c}^{(\Lambda)}$. Hence higher scales of chiral symmetry breaking will be expected, e.g. $\Lambda \geq \Lambda_{c}=2647 \mathrm{GeV}$ for $\beta \geq \beta_{\min }=0.726835$.

A complete determination of the $\beta-\alpha^{(\Lambda)}$ criticality depend on the value of $B / A$ which is fixed by the IR boundary condition and in present scheme by the value of $\xi$. In addition, more exact consideration of non-linearity of $\Sigma(\tau)$ in small $\tau$ region is also necessary. These facts also imply that we can not make a completely precise prediction of the scale of chiral symmetry breaking unless we have precisely known the IR behavier of the gauge coupling constant and the non-linearity behavier of $\Sigma(\tau)$ either from a fundamental theory or from phenomenology. However, by means of the exact solutions of the linearized S-D equation of fermion self-energy, we have given the general feature of the $\beta-\alpha^{(\Lambda)}$ criticality curve which do not depend on the details of the IR behavier of the theory. In particular, we see from Fig. 1 that in the extreme UV region where $\alpha^{(\Lambda)} \approx 0$, the four curves (a), (b), (c) and $(\mathrm{d})$ with different values of the IR parameter $\xi$ almost coincide with each other. On the other hand, once the IR parameter $\xi$ is given separately, we may make quite definite estimations of the scales of chiral symmetry breaking. All these show obvious advantages of the approach of exact solutions over general numerical methods.

The paralell analyses may be applied to the top-quark condensate scheme of electroweak symmetry breaking if we identify $G=S U_{c}(3)$ and the $\psi$-field with the top-quark field. If the six quark flavors are included in calculation of $\beta_{0}$ we will have $b=4 / 7$. Similar results to the ones in TC theory can be obtained except that the scale of chiral symmetry breaking would be much lower. In the case of pure gauge interactions, i.e. $B / A \rightarrow \infty$, we have $\xi=1.40025$ and the corresponding zero-momentum gauge coupling $\alpha(0)=4 \pi / 7 \ln \xi=4.9208$ which is bigger than the result from numerical analyses [11] if it is identified with the critical gauge coupling for chiral symmetry breaking. 
The difference could be attributed to the method and approximation used here. To estimate the scale of chiral symmetry breaking, let us consider the case of $\xi=2$. A similar $\beta-\alpha(\Lambda)$ criticality curve to the curve (b) in Fig.1 will be obtained but with $\beta=\beta_{\min }=0.570637$ at $\alpha^{(\Lambda)}=\alpha_{c}^{(\Lambda)}=0.58046$ which corresponds to the smallest scale of chiral symmetry breaking $\Lambda_{c}=408 \mathrm{GeV}$ if we take the scale parameter $\mu$ to be the mass of $Z$-boson $M_{Z}=91.187 \mathrm{GeV}$ [16]. Since the resulting $\Lambda_{c} \ll \Lambda_{\text {top }}$, the momentum cut-off in the bubble diagram of the four-fermion interactions, which is up to $10^{15} \mathrm{GeV}$ (the grand unification scale) in the simplest top-quark condensate scheme [6] and also at least above $5 \times 10^{3} \mathrm{GeV}$ even in an exteded version of the scheme including the fourth generation of fermions [17], the chiral symmetry breaking will happen actually at a point near $\left(\alpha^{(\Lambda)}, \beta\right)=(0,1)$ in the $\beta-\alpha^{(\Lambda)}$ criticality curve. This only reproduces the fact that the top-quark condensate scheme is essentially a NJL model with some small corrections from the color (and in addition, electroweak) interactions. Certainly, by means of the S-D equation of fermion self-energy and the linearization approximation presented in this paper, we could deal with the corrections to the fermion mass from the color gauge interactions. This approach could become an alternative one of the renormalization group analyses extensively used in pursuing this kind of problem $[5,6,18,19]$.

\section{References}

[1] S.Weinberg, Phys. Rev. D19(1979)1277; L.Susskind, Phys.Rev. D20(1979)2619.

[2] S.Dimopoulos and L.Susskind, Nucl.Phys.B155(1979)237; E.Eichten and K.Lane, Phys.Lett. 90B(1980)125.

[3] Y.Nambu, in New Theories in Physics, Proceedings of the XI International Symposium on Elementary Particle Physics, Kazimierz, Poland, 1988, edited by Z.Ajduk, S.Porkorski and A.Trautman (World Scientific, Singarpore, 1989).

[4] V.A.Miransky, M.Tanabashi and K.Yamawaki, Phys. Lett. B221(1989)177.

[5] W.J.Marciano, Phys. Rev. Lett. 62(1989)2793.

[6] W.A.Bardeen, C.T.Hill and M.Lindler, Phys. Rev. D41 (1990)1647.

[7] K.Lane, Phys. Rev. D10(1974)2605; H.D.Politzer, Nucl. Phys. B117(1976)397.

[8] P.I.Fomin, V.P.Gusynin, V.A.Miransky and Yu.A.Sitenko, Rev. Nuovo Cimento 6(1983)1; K.Higashijima, Phys. Rev. D29(1984)1228.

[9] V.A.Miransky, Phys. Lett. B165(1985)401.

[10] C.Leung, S.Love and W.A.Bardeen, Phys. Rev. Lett. 56(1986)1230; Nucl. Phys. B273(1986)649.

[11] D.Atkinson and P.W.Johnson, Phys.Rev.D37(1988)2290; 2296; D.Atkinson, P.W.Johnson and K.Stam, Phys. Rev. D37(1988)2996.

[12] D.Atkinson and P.W.Johnson, Phys. Rev.D41(1990)1661; H.J.Munczek and D.W.Mckay, Phys. Rev. D39(1989)888; ibid., D42(1990)3548; D.Atkinson, H.de Groot and P.W.Johnson, Phys. Rev. D43(1991)218; A.G.Williams, G.Krein and C.D.Roberts, Ann. Phys. 210(1991)464.

[13] T.Appelquist, M.Soldate, T.Takeuchi and L.C.R.Wijewardhana, in: Proc. 12th Johns Hopkins Workshop on Current Problems in Particle Theory (1988)197. 
[14] B.R.Zhou, J. Math. Phys., 38(1997)809.

[15] Y.Nambu and G.Jona-Lasinio, Phys. Rev. 122(1961)345; ibid. 124(1961)246.

[16] Particle Datd Group, Phys. Rev. D50(1994)1173.

[17] B.R.Zhou, Phys. Rev. D47(1993)2656;D48(1993)4484(E); ibid.D47(1993)5038; D50(1994) 578 .

[18] W.J.Marciano, Phys. Rev.D41(1990)219; M.Suzuki, Phys. Rev. D41(1990)3457; M.Luty, Phys. Rev. D41(1990)2893; C.T.Hill, M.A.Luty and E.A.Paschos, Phys. Rev. D43(1991)3011; R.F.Lebed and M.Suzuki, Phys. Rev D45(1992)1744; S.F.King, Phys. Lett. B281(1992)295.

[19] B.R.Zhou, Commun.Theor.Phys. $26 \quad$ (1996) 97; 26(1996)235; 27(1997)77; 28(1997)79. 